\title{
Intervenções fisioterapêuticas em pacientes queimados na Unidade de Terapia
}

\section{Intensiva: revisão bibliográfica}

\author{
Physiotherapeutic interventions in burn patients in the Intensive Care Unit: literature review \\ Intervenciones fisioterapéuticas en pacientes quemados en la Unidad de Cuidados Intensivos: \\ revisión bibliográfica
}

Recebido: 28/10/2021 | Revisado: 07/11/2021 | Aceito: 10/11/2021 | Publicado: 17/11/2021

\author{
Deyse Santos da Silva \\ ORCID: https://orcid.org/0000-0002-5754-3327 \\ Centro Universitário do Norte, Brasil \\ E-mail: deyses136@gmail.com \\ Bárbara Lira Bahia Mendes \\ ORCID: https://orcid.org/0000-0002-8363-3211 \\ Centro Universitário do Norte, Brasil \\ E-mail: fisio.barbara@hotmail.com
}

\begin{abstract}
Resumo
Introdução: Queimaduras são lesões que podem ser superficiais acometendo apenas a pele bem como comprometendo camadas mais profundas da pele podendo chegar ao osso. Objetivo: O principal objetivo da seguinte revisão é mostrar a importância e relevância do fisioterapeuta na tratativa com pacientes queimados em âmbito hospitalar. Metodologia: O seguinte artigo trata-se de uma revisão bibliográfica sistemática considerando artigos dos períodos de 2012 a 2021 , nas bases de dados: literatura Latino Americana e do Caribe em Ciências da Saúde (LILACS), U.S National Institute of Health (PubMed), Scientific Eletronic Library Online (SciElo). Resultados e discussão: O profissional de fisioterapia esta inserido em uma unidade de terapia intensiva para compor uma equipe multidisciplinar a fim de proporcionar benefícios a todos os pacientes ali presentes com planos de tratamento individualizado. Conclusão: Sendo assim, com o intuito de devolver funcionalidade ao paciente, com condutas precoces para retirada do mesmo do leito a fim de inibir as deformidades, contraturas e patologias associadas.
\end{abstract}

Palavras-chave: Fisioterapia; Queimados; Unidade de Terapia Intensiva.

\begin{abstract}
Introduction: Burns are injuries that can be superficial, affecting only the skin, as well as compromising layers of the skin being able to reach the bone. Objective: The main objective of the following review is to show the importance and relevance of the physiotherapist in the treatment of burn patients in the hospital environment. Methods: The following article is the following article is a literature review systematic considering articles from 2012 to 2021 in the databases: Latin American and Caribbean Literature on Health Sciences (LILACS), US National Institute of Health(PubMed), Scientific Electronic Library Online (SciElo). Results and discussion: The physiotherapy professional is inserted in an intensive care unit to compose a multidisciplinary team in order to provide benefits to all patients there with individualized treatment plant s. Conclusion: Therefore, in order to return functionality to the patient, either early conduct to remove him from the bed for the purpose of inhabit deformities, contractures and associated pathologies.
\end{abstract}

Keywords: Physiotherapy; Burned; Intensive Care Unit.

\section{Resumen}

Introducción: las quemaduras son lesiones que pueden ser superficiales, afectando solo la piel, además de comprometer capas más profundas de piel y llegar al hueso. Objetivo: El objetivo principal de la siguiente revisión es mostrar la importancia y relevancia de los fisioterapeutas en el tratamiento de pacientes quemados en entornos hospitalarios. Metodología: El siguiente artículo es una revisión de la literatura sistemático considerando artículos de los períodos 2012 a 2021, em las bases de datos: Literatura Latinoamericana y del Caribe em Ciencias de la Salud (LILACS), Instituto Nacional de Salud de los Estados Unidos (PubMed), Librería Electrónica Científica em Línea (SciElo). Resultados y discusión: El profesional de fisioterapia se inserta en una unidad de cuidados intensivos para componer un equipo multidisciplinario con el fin de brindar beneficios a todos los pacientes allí presentes con planes de tratamiento individualizados. Conclusión: Por tanto, para devolver la funcionalidad al paciente, con conductas tempranas para sacar al paciente de la cama con el fin de inhibir deformidades, contracturas y patologías asociadas.

Palabras clave: Fisioterapia; Quemado; Unidad de Terapia Intensiva. 


\section{Introdução}

Nas últimas décadas as Unidades de Terapia Intensiva (UTI) têm se tornado uma concentração não somente de pacientes críticos e te tecnologia avançada, mas, também de uma equipe multiprofissional experiente com competências especificas. Existem no Brasil mais de 1.500 unidades de terapia intensiva cadastradas na Associação de Medicina Intensiva Brasileira (AMIB) e com diferentes características e possivelmente, com fisioterapeutas ali trabalhando. Nesta revisão bibliográfica terá como público alvo, adultos que passaram ou ainda estão em Unidades de Terapia Intensiva (UTI) no âmbito do Brasil.

Apresentando os processos fisiológicos do sistema tegumentar, cuidados da fisioterapia para com esses pacientes e mostrando que a mesma faz parte de um processo de reabilitação, contribuindo em questões biopsicossociais, respiratórias, motoras e ainda na atuação dermato-funcional em indivíduos acometidos por queimaduras. De acordo com a Sociedade Brasileira de Queimaduras (2012) estimasse que no Brasil ocorressem em torno de 1.000 .000 acidentes com queimadura por ano sendo que destes 100.000 acidentados procuram atendimento hospitalar e por cerca de 2.500 irão vir a óbito por conta das lesões. E conforme a Organização Mundial de Saúde (2012) estimasse que 322.000 pessoas morram a cada ano em decorrência de queimaduras relacionadas ao fogo.

De acordo com Bernardo, Santos e Silva (2019), a pele é o maior órgão humano onde a mesma funciona como empecimento e protetora contra agentes externos infecciosos, patologias e possíveis agressões ambientais, além de regular a temperatura corporal possuindo três camadas: epiderme, derme e hipoderme.

A queimadura pode ser definida como trauma capaz de ocasionar variadas lesões que, de acordo com o nível, pode levar o enfermo a óbito, tais como, hiperemia restrita à área queimada, alterações celulares e imunológicas decorrentes do insulto, envolvimento das vias respiratórias e ocorrência de traumatismos associados (apud Borges, 2012, p. 2).

As queimaduras podem ser classificadas de acordo com a extensão da superfície corpórea queimadas, calculada em porcentagem total da área queimada (ATSQ). É possível, ainda, classificar queimaduras quanto à profundidade destas, as quais podem ser de primeiro, segundo ou terceiro grau. Quanto à extensão, o indivíduo pode ser classificado em: pequeno queimado, ou de pequena gravidade; médio queimado ou de média gravidade e grande queimado ou de grande gravidade (Almeida JWF, 2013 apud Santos JN, 2013).

A classificação quanto à profundidade da queimadura é observada em graus (I,II,III, IV). As queimaduras de primeiro grau restringem-se a epiderme, são superficiais e não apresentam alterações hemodinâmicas ou clínicas importantes. As de segundo grau atingem a epiderme e parte da derme, apresentam bolhas ou flictenas. As de terceiro grau são consideradas graves, pois atingem toda a epiderme, derme, podendo em muitos casos, lesar a tela subcutânea, tecido muscular e ósseo. A queimadura de quarto grau é a queimadura elétrica, que envolve a destruição completa de todos os tecidos. O prognóstico, nesse caso, é incerto, sendo necessária uma extensa excisão cirúrgica ou, até mesmo, amputação (apud Pierini, 2014, p. 2).

Segundo o ministério da saúde (2012) a região norte é aparentemente, dentre todas as regiões do país, a que contribui com os menores números nas estatísticas sobre o assunto, representando aproximadamente 7,5\% das internações em virtude desse agravo, tendo também a menor taxa de mortalidade da federação (0,90\%). O Para, é o estado da região norte com os piores indicadores em relação às queimaduras.

Pinho et al (2016) dizem que os traumas por queimaduras trazem consequências graves ao corpo humano visto que acarreta danos metabólicos, podem afetar órgãos e sistemas que produzem danos imediatos, deixando sequelas físicas e emocionais ao paciente queimado e sua família além, das incapacidades funcionais que podem seguir como sequelas. 
Os principais objetivos serão mostrar e descrever a relevância da fisioterapia em pacientes queimados em Unidades de Terapia Intensiva (UTI), elucidando aspectos anatômicos e fisiológicos de forma a evidenciar as condutas fisioterapêuticas baseadas em evidencias.

\section{Metodologia}

O estudo é caracterizado como revisão bibliográfica sistemática, descritivo e explicativo com uma abordagem qualitativa e demonstrativa. Utilizando as 8 etapas da revisão sistemática que são: formular a questão da revisão, definir critérios de inclusão e exclusão, estratégias de busca, seleção de estudos, avaliação da qualidade dos estudos, extração de dados, analise e apresentação de dados e interpretação dos resultados.

Também foram definidos os seguintes descritores indexados no DeCS (Descritores em Ciências da Saúde): fisioterapia, estatísticas, complicações, queimados e unidade de terapia intensiva (UTI). Utilizando as bases de dados: literatura Latino Americana e do Caribe em Ciências da Saúde (LILACS), U.S National Institue of Health (PubMed), Scientifc Eletronic Libbrary Online (SciElo). A busca pelos artigos científicos foi realizada entre os meses de abril a setembro de 2021.

Inicialmente foram selecionados 66 artigos pelos critérios de inclusão: artigos disponíveis no idioma inglês e português e publicado entre os anos de 2012 e 2021. Após aplicar os critérios de exclusão que foram: artigos que se direcionavam a tratativas sobre pacientes fora do âmbito hospitalar, resumos, artigos incompletos, monografias, teses e artigos de anos anteriores a 2012. Após a leitura e aplicados os critérios foram selecionados 12 artigos através do tema, resumo e palavras-chave para a composição do presente trabalho.

Os resultados submetidos e analisados foram tabulados e expostos em forma de tabela, na tabela 1 será demonstrada a síntese dos artigos, na Tabela 2 os dados negativos e positivos referentes a intervenções fisioterapêuticas, na figura 1 o fluxograma do processo de seleção dos artigos.

Figura 1 - Fluxograma de seleção dos artigos.

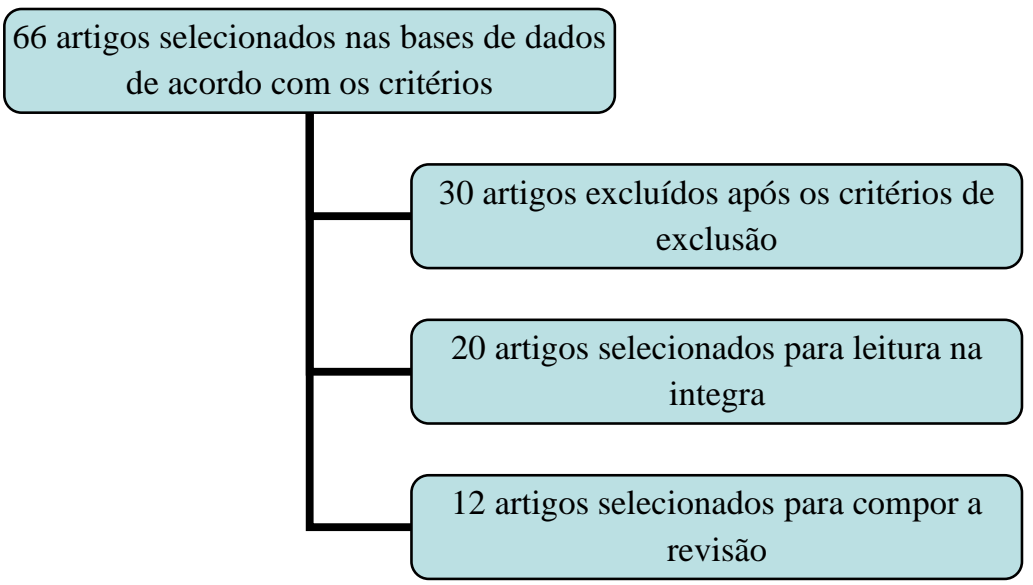

Fonte: Autores. 
Tabela 1 - síntese dos artigos selecionados.

\begin{tabular}{|c|c|c|c|}
\hline AUTOR/ANO & OBJETIVO & METODOLOGIA & CONCLUSÃO \\
\hline $\begin{array}{c}\text { (Cruz, et al., } \\
\text { 2012) }\end{array}$ & $\begin{array}{l}\text { Demonstrar os índices estatísticos de } \\
\text { acidentes por queimadura no Brasil, } \\
\text { identificando o perfil epidemiológico dos } \\
\text { indivíduos que sofrem esses traumas, } \\
\text { identificando as causas e fatores. }\end{array}$ & $\begin{array}{l}\text { Trata-se de uma revisão bibliográfica, } \\
\text { secundaria retrospectiva e descritiva. } \\
\text { Sendo considerados periódicos nacionais, } \\
\text { utilizando os seguintes descritores: } \\
\text { fisioterapia, queimadura, causas das } \\
\text { queimaduras, epidemiologia das } \\
\text { queimaduras, juntos e separados. }\end{array}$ & $\begin{array}{l}\text { Conclui-se que o sexo masculino é o mais afetado por } \\
\text { acidentes com queimaduras, em todas as faixas etárias } \\
\text { exceto crianças de } 0 \text { a } 4 \text { anos, que são as mais } \\
\text { acometidas por queimaduras. A média de idade dos } \\
\text { adultos foi de } 25 \text { a } 26 \text { anos como causador principal o } \\
\text { álcool. Evidenciando que o domicilio é o local de maior } \\
\text { ocorrência de acidentes. }\end{array}$ \\
\hline $\begin{array}{l}\text { (Morais, et al., } \\
\text { 2015) }\end{array}$ & $\begin{array}{l}\text { Descrição de condutas fisioterapêuticas } \\
\text { utilizadas em um paciente em grande } \\
\text { queimado durante a internação hospitalar. }\end{array}$ & $\begin{array}{l}\text { Relato de caso, se tratando de um } \\
\text { paciente de } 17 \text { anos do sexo masculino } \\
\text { que deu entrada no Centro de tratamento } \\
\text { que Queimados do Hospital } \\
\text { Metropolitano de Urgência e Emergência } \\
\text { de Ananindeua PA. }\end{array}$ & $\begin{array}{l}\text { A correção do paciente no leito é fundamental para } \\
\text { evitar deformidades, fibrose, rigidez, contraturas, úlceras } \\
\text { e edemas. A proposição do plano fisioterapêutico retarda } \\
\text { não apenas os itens a cima citados, podendo ser inclusos } \\
\text { complicações pulmonares, por isso devendo ser precoce } \\
\text { e continua a atuação do fisioterapeuta. }\end{array}$ \\
\hline $\begin{array}{l}\text { (Comini, et al., } \\
\text { 2017) }\end{array}$ & $\begin{array}{l}\text { Analisar o perfil epidemiológico de } \\
\text { pacientes idosos internados na Unidade de } \\
\text { Tratamento de Queimados do Hospital Padre } \\
\text { Albino em Catanduva. }\end{array}$ & $\begin{array}{l}\text { Estudo transversal baseado em analises } \\
\text { dos prontuários e do livro de registro da } \\
\text { Unidade de Terapia de Queimados, sendo } \\
\text { feito a coleta entre março de } 2012 \text { e } \\
\text { março de } 2013 \text { de idosos que passaram } \\
\text { pela unidade. Coletados dados como: } \\
\text { sexo, idade, comorbidades, agente } \\
\text { agressor e profundidade da lesão. }\end{array}$ & $\begin{array}{l}\text { O conhecimento do perfil epidemiológico dos pacientes } \\
\text { vem a ser de extrema importância para que o serviço } \\
\text { hospitalar prestado seja com menos risco e com } \\
\text { condições mais comuns para evitar intercorrências. }\end{array}$ \\
\hline $\begin{array}{l}\text { (Santiago, et al., } \\
\text { 2018) }\end{array}$ & $\begin{array}{l}\text { Confrontar dados e reafirmar a relevância da } \\
\text { fisioterapia no tratamento de pacientes } \\
\text { queimados. }\end{array}$ & $\begin{array}{l}\text { Caracterizada como revisão de literatura } \\
\text { descritiva, cujo levantamento de } \\
\text { bibliográfica foi feito na base de dados } \\
\text { Scielo e Lilacs pelos descritores: } \\
\text { fisioterapia; queimaduras; unidade de } \\
\text { terapia intensiva. }\end{array}$ & $\begin{array}{l}\text { Sendo a fisioterapia de grande valia em todo o } \\
\text { acompanhamento ambulatorial, acrescentando condutas } \\
\text { importantes em todas essas fases, atuando também de } \\
\text { forma complementar as cirurgias realizadas. }\end{array}$ \\
\hline $\begin{array}{l}\text { (Ferreira, et al., } \\
\text { 2020) }\end{array}$ & $\begin{array}{l}\text { Analisar dificuldades enfrentadas pelos } \\
\text { profissionais de enfermagem na assistência } \\
\text { de pacientes queimados, verificar e } \\
\text { identificar problemas de cunho } \\
\text { familiar/enfermeiro. }\end{array}$ & $\begin{array}{l}\text { Estudo descritivo, exploratório com } \\
\text { abordagem qualitativa, feito à coleta de } \\
\text { dados com profissionais de Hospital } \\
\text { referencia em um municio do interior do } \\
\text { nordeste. }\end{array}$ & $\begin{array}{l}\text { Foram apontadas diversas dificuldades enfrentadas pela } \\
\text { equipe, havendo a necessidade de mais profissionais } \\
\text { para uma atuação integral. }\end{array}$ \\
\hline $\begin{array}{l}\text { (Giordani, et al., } \\
\text { 2016) }\end{array}$ & $\begin{array}{l}\text { Identificar as principais complicações } \\
\text { durante o tratamento hospitalar do paciente } \\
\text { queimado. }\end{array}$ & $\begin{array}{l}\text { Trata-se de uma revisão integrativa de } \\
\text { literatura de artigos disponibilizados nas } \\
\text { plataformas: Scielo, PubMed e Lilacs a } \\
\text { partir dos descritores: queimaduras, } \\
\text { complicações e enfermagem. }\end{array}$ & $\begin{array}{l}\text { Por concluído que as principais complicações dos } \\
\text { pacientes queimados vêm a serem a sepse, infecção, } \\
\text { distúrbios sanguíneos, distúrbios respiratórios, distúrbios } \\
\text { cardíacos, distúrbios renais e transtornos emocionais. }\end{array}$ \\
\hline $\begin{array}{l}\text { (Souza, et al., } \\
2015)\end{array}$ & $\begin{array}{l}\text { Relatar a ocorrência e o tratamento de um } \\
\text { caso de queimadura atingindo toda a face do } \\
\text { individuo. }\end{array}$ & $\begin{array}{l}\text { Trata-se de um relato de caso de uma } \\
\text { criança vitima de incêndio domestico que } \\
\text { foi encaminhada para o Centro de } \\
\text { Tratamento de Queimaduras da Bahia. }\end{array}$ & $\begin{array}{l}\text { O relato confirma que a utilização do couro cabeludo a } \\
\text { fins estéticos tem sido bastante favorável, seguro e com } \\
\text { vantagens para queimaduras em face. }\end{array}$ \\
\hline $\begin{array}{l}\text { (Santana, et al., } \\
\text { 2012) }\end{array}$ & $\begin{array}{l}\text { Analisar a importância da fisioterapia na } \\
\text { reabilitação de pacientes com queimaduras } \\
\text { com protocolos de avaliação antes e depois } \\
\text { da intervenção fisioterapêutica. }\end{array}$ & $\begin{array}{l}\text { Estudo de intervenção e de campo, com } \\
\text { natureza qualitativa e quantitativa, } \\
\text { amostra composta por } 30 \text { voluntários } \\
\text { analisando aspectos: dor de reparo } \\
\text { cicatricial, agente causador, edema, grau e } \\
\text { extensão da queimadura. }\end{array}$ & $\begin{array}{l}\text { Os parâmetros clínicos dos pacientes comparados antes e } \\
\text { após a fisioterapia apresentam valores significativos de } \\
\text { melhora confirmando a importância da intervenção do } \\
\text { profissional. }\end{array}$ \\
\hline $\begin{array}{l}\text { (Santos, et al., } \\
\text { 2017) }\end{array}$ & $\begin{array}{l}\text { Descrever o perfil epidemiológico dos } \\
\text { adultos internados em um centro de } \\
\text { tratamento de referencia em queimaduras. }\end{array}$ & $\begin{array}{l}\text { Estudo descritivo, transversal, com } \\
\text { abordagem quantitativa realizado em } \\
2016 \text { no Centro de Tratamento de } \\
\text { Queimados de Fortaleza. }\end{array}$ & $\begin{array}{l}\text { Os achados foram: produtos inflamáveis são os } \\
\text { principais causadores, os indivíduos possuem baixo } \\
\text { nível socioeconômico sendo classificados como médio e } \\
\text { grande queimado, onde o tronco foi à região do corpo } \\
\text { mais afetada. }\end{array}$ \\
\hline $\begin{array}{l}\text { (Felix, et al., } \\
\text { 2014) }\end{array}$ & $\begin{array}{l}\text { Descrever a melhor informação para a } \\
\text { tomada de decisão e identificar o maior } \\
\text { numero possível de estudos relacionados à } \\
\text { relevância fisioterapêutica em pacientes } \\
\text { queimados. }\end{array}$ & $\begin{array}{l}\text { Realizada uma revisão sistemática, } \\
\text { retrospectiva e descritiva. Disponível nas } \\
\text { bases de dados: Lilacs, Scielo no idioma } \\
\text { português. }\end{array}$ & $\begin{array}{l}\text { Obtendo como conclusão que o fisioterapeuta atua em } \\
\text { todas as consequências da queimadura, não apenas no } \\
\text { diz respeito da motricidade e respiratória bem como no } \\
\text { âmbito psicológico dos pacientes. }\end{array}$ \\
\hline $\begin{array}{l}\text { (Queiroz, et al., } \\
\text { 2015) }\end{array}$ & $\begin{array}{l}\text { Identificar o perfil dos indivíduos que } \\
\text { desistiram do tratamento ambulatorial de } \\
\text { queimados no Hospital de Sorocaba. }\end{array}$ & $\begin{array}{l}\text { Trata-se de um estudo exploratório, } \\
\text { descritivo, transversal, quantitativo com } \\
\text { pacientes adultos que sofreram } \\
\text { queimaduras tendo abandonado o } \\
\text { tratamento ambulatorial. }\end{array}$ & $\begin{array}{l}\text { A avalição trouxe dados que as pessoas desistentes eram } \\
\text { economicamente ativas porem, ficando impossibilitadas } \\
\text { de prosseguir suas funções que contribuiu para a } \\
\text { desistência, aliado a pobreza de informação. }\end{array}$ \\
\hline $\begin{array}{l}\text { (Lopes, et al., } \\
\text { 2014) }\end{array}$ & $\begin{array}{l}\text { Averiguar como a atuação terapêutica } \\
\text { contribui na reabilitação física de pacientes } \\
\text { queimados favorecendo a promoção da } \\
\text { funcionalidade e redução dos danos } \\
\text { causados. Identificando os principais } \\
\text { recursos e técnica para a reabilitação dos } \\
\text { pacientes vitima de queimaduras. }\end{array}$ & $\begin{array}{l}\text { Trata-se de uma revisão bibliográfica de } \\
\text { abordagem qualitativa considerando os } \\
\text { aspectos descritivos. }\end{array}$ & $\begin{array}{l}\text { Há um efeito benéfico no que tange a abordagem } \\
\text { terapêutica atuando como potencializador e facilitador } \\
\text { da independência do individuo, fazendo uso de treino de } \\
\text { atividades básicas da vida diária, avaliação e } \\
\text { fortalecimento como forma geral. Podendo ser inserido } \\
\text { em todas as fases no tratamento de pessoas acometidas } \\
\text { por queimaduras. }\end{array}$ \\
\hline
\end{tabular}

Fonte: Dados da pesquisa (2021). 


\section{Resultados e Discussão}

De acordo com todos os autores e artigos a presente revisão mostrou a importância de se ter um profissional de fisioterapia em uma unidade hospitalar para que a redução da taxa de mortalidade seja efetiva assim como a mobilização precoce no leito seja realizada, visto que na atualidade o mesmo acarreta grande parte do agravo de doenças associadas à longa permanência do individuo em uma unidade hospitalar.

Após as analises encontradas podemos apontar as lesões por queimaduras traumas extensos com especificidades únicas a serem tratadas, às mesmas devendo ter manejos específicos com intervenções medicas, fisioterapêuticas, da enfermagem e de toda a equipe multidisciplinar para a não proliferação de agentes infecciosos e patologias adjacentes sendo assim não acarretando o aumento da lesão.

O fisioterapeuta terá por objetivo precoce ajudar no processo cicatricial, reduzir e/ou inibir no processo de aparecimento das escaras e úlceras de pressão que podem surgir com o tempo em que o paciente fica acamado na unidade hospitalar, no que diz respeito ao trato respiratório e sistema musculoesquelético o profissional poderá realizar intervenções e técnicas respiratórias, motoras e manuais trazendo um maior conformo ao paciente, aumentando o nível de dependência e funcionalidade do paciente.

Tabela 2 - Efeitos negativos e positivos referentes a intervenções fisioterapêuticas.

\begin{tabular}{ll}
\hline Efeitos deletérios sem intervenção da fisioterapia & \multicolumn{1}{|c}{$\begin{array}{c}\text { Efeitos benéficos com intervenções } \\
\text { fisioterapêuticas }\end{array}$} \\
\hline Perda de habilidades. & $\begin{array}{l}\text { Prevenir perda de massa muscular. } \\
\text { Diminuição das lesões por pressão. }\end{array}$ \\
\hline $\begin{array}{l}\text { Aumento do ciclo cardíaco. } \\
\text { Desenvolvimento de doenças trombóticas. }\end{array}$ & $\begin{array}{l}\text { Prevenção de complicações pulmonares. } \\
\text { Infecções no trato respiratório. }\end{array}$ \\
$\begin{array}{l}\text { Maior taxa de mortalidade e dependência de } \\
\text { terceiros. }\end{array}$ & $\begin{array}{l}\text { Maior taxal de altas definitivas e independência } \\
\text { funcional. }\end{array}$ \\
\hline
\end{tabular}

Fonte: Dados da pesquisa (2021).

Os dados contidos no presente quadro faz o levantamento de principais benefícios acarretados com intervenções fisioterapêuticas no âmbito hospitalar e efeitos deletérios sem as intervenções do profissional. Podendo ser observado a retrogressão do paciente queimado sem o profissional de fisioterapia para realizar o devido acompanhamento.

De acordo com Giordani et al (2016) no atendimento hospitalar a condição clinica do paciente queimado podem evoluir para septicemia, insuficiência respiratória, mudanças metabólicas, alterações cardíacas, renais e gastrointestinais.

Para Schott et al. (2014) a fisiopatologia da lesão por queimadura se da pela destruição da integridade capilar e vascular, em razão de seus efeitos serem locais e sistêmicos. O comprometimento do tecido vai depender da intensidade da exposição térmica, das características da área queimada e das reações locais e sistêmicas.

Nesse contexto, Ryan et al. (2012) diz que no processo fisiológico, primeiramente ocorre necrose eosinofílica e após, edema e intensa congestão hemorrágica, com os tecidos evoluindo do esbranquiçado para escara acinzentada, ficando preta logo em seguida. Inicia-se o tecido de granulação 10 dias depois; em cerca de três semanas, há propagação fibroblástica e cicatricial, com começo das estenoses.

Nossos corpos tem um sistema especial para combater os diversos agentes infecciosos e tóxicos aos quais estamos continuamente expostos. Os leucócitos (glóbulos brancos) são as unidades moveis do sistema de proteção do corpo. Eles são formados na medula óssea e no tecido linfático, sendo transportados no sangue para as áreas de inflamação, a fim de proporcionar uma defesa rápida e potente contra os agentes infecciosos (Guyton, 2017, p 207). 
Ainda segundo Guyton (2017) quando há uma lesão tecidual, diversas substancias são liberadas, causando alterações secundarias no tecido. Essas substâncias aumentam o fluxo sanguíneo local e a permeabilidade dos.

Um estudo de Salgado et al. (2013) diz que os processos de recomposição epidérmica e a própria capacidade do organismo reconstruir-se podem ser alcançados com maior rapidez por meio de cuidados básicos como: desbridamento, limpeza e higiene local. Esse processo tem por objetivo e finalidade o fechamento primário da ferida e cicatrização por segunda intenção.

O Ministério da Saúde (2015) relata que o profissional de fisioterapia aparece como componente de uma equipe multidisciplinar dentro da unidade de terapia intensiva (UTI), estudos recentes mostram a importância do trabalho deste profissional na redução do tempo de internação e na qualidade de vida pós-alta dos pacientes. Embora a maioria das pessoas desconheça a presença do profissional de fisioterapia nas unidades de tratamento intensivo, no Brasil desde a década de setenta seu trabalho no ambiente é obrigatório e muito importante, além de complementar às demais áreas que desenvolvem seu trabalho na unidade de terapia intensiva (UTI).

Para Carvalho et al (2013) a fisioterapia tem como principal objetivo preservar, melhorar e restaurar quando necessário à capacidade funcional dos pacientes, diminuindo os riscos do repouso prolongado e garantindo independência física.

Reis et al (2014) diz que para a avaliação inicial são realizadas medidas de goniometria, força muscular, avaliação da sensibilidade, avaliação do edema e quantificação subjetiva da dor caso seja possível.

Para Costa et al. (2014) o profissional de fisioterapia presente dentro de uma unidade de terapia intensiva (UTI) pode atuar na prevenção de deformidades, manutenção da mobilidade articular e restituição da função, posicionamento no leito adequado, estimulação da movimentação precoce, o uso de órteses dependendo da necessidade do paciente, controle da cicatrização, redução de edema e ainda treinos de atividades da vida diária. Todos esses objetivos que se encaixam dentro de um plano de tratamento para a reabilitação do paciente queimado.

Outra abordagem de acordo com George et al (2014) é o controle cicatricial, a formação de tecido cicatricial é uma resposta natural para o fechamento da ferida. Uma cicatriz hipertrófica é vermelha, elevada e inelástica, contém um número aumentado de fibroblastos, quando comparada a pele normal. Além de não ser esteticamente atraente, a cicatriz hipertrófica pode limitar a competência funcional ao restringir a amplitude de movimento da articulação.

Segundo Busnardo et al. (2014) a cinesioterapia é a movimentação ativa ou passiva, sendo a passiva realizada apenas quando o paciente/individuo não estiver em possibilidades para ativamente cumprir os movimentos propostos. Os mesmos devem estimular os segmentos afetados, podendo ser executado varias vezes ao dia de forma lenta, suave e progressiva de forma individualizada com o objetivo de completar todo o arco do movimento atingindo a amplitude de movimento esperada para cada articulação. As articulações das extremidades também devem ser movimentadas a menos que tenham contraindicação como uma fratura exposta.

Conforme Teixeira et al. 2014, quando o paciente já se encontra em fase ambulatorial certamente não apresentará nenhuma ulcera ou escara aberta podendo ser iniciado as funções manuais tais como as de preensão com certo grau de força e precisão, permitindo ainda para o profissional uma ampla variedade de exercícios que podem ser executados.

Rivrs (2014 apud Jordan, 2014) dizem que os exercícios básicos utilizados são os ativos livres, os ativos assistidos, os isométricos, os de estiramentos e os de coordenação, todos tendo como objetivo a prevenção de deformidades.

Outro aspecto a ser enfatizado é a independência nas atividades de vida diária, habilidades como alimentar-se, vestirse, arrumar-se e banhar-se devem ser reforçadas como parte de uma rotina normal. A identificação inicial de movimentos anormais ajuda os pacientes a entender suas necessidades e trás oportunidades para reaprender padrões normais de movimento. 
Praticas as atividades básicas da vida com itens de cuidado pessoal trazidos de casa pode alimentar uma atitude positiva em relação aos sentimentos sobre as capacidades pessoais (Arruda et al. 2007).

\section{Considerações Finais}

Mediante o exposto é necessário salientar que a fisioterapia pode e deve ser iniciada de forma precoce a fim de diminuir as proliferações das lesões, desnutrição do paciente, novas infecções, sepse e as taxas de mortalidade que uma permanência prolongada no leito pode ocasionar.

O presente artigo permitiu compreender a importância de um profissional de fisioterapia compondo o quadro de uma equipe multidisciplinar em uma unidade hospitalar onde a presença do mesmo acarreta mudanças e benefícios biopsicossociais ao paciente queimado. A avaliação beira leito do paciente, bem como saber diferenciar a etiologia, os tipos e graus de queimadura é insubstituível para que o profissional trace condutas adequadas para o individuo levando em consideração suas necessidades e particularidades anatômicas, fisiológicas e sociais.

Desta forma, a notabilidade do mesmo em âmbito hospitalar dedicando-se as lesões por queimaduras desde atenção primaria quando há necessidade de informar a sociedade como um todo, os cuidados domésticos que se deve ter a cerca do tema bem como na atenção terciaria, onde foi o foco do artigo, cuidando e tratando das lesões associadas vinculadas com condutas fisioterapêuticas a fim de devolver a funcionalidade das vitimas de queimaduras para que as mesmas possam voltar ao âmbito social.

Para fins de sugestões finais, de suma importância às discussões do tema abordado em sociedade que trabalham em nível de notabilidade primaria onde é disseminadas informações a população a cerca de como prevenir possíveis lesões domesticas de queimaduras, de como realizar os primeiros socorros a um individuo vitima de queimadura grave, informações a trabalhadores que lidam diariamente com materiais inflamáveis, podem ser de grande valia para evitar crescimentos de internações e mortalidades pela causa.

\section{Agradecimentos}

Agradecemos a todos que direta ou indiretamente contribuíram para a realização e sucesso do artigo.

\section{Referências}

Cruz, B. F., Cordovil, P. B. L. \& Batista, K. N. M. (2012). Perfil epidemiológico de pacientes que sofreram queimaduras no Brasil: revisão de literatura. Rev Bras Queimaduras, 11(4):246-50 http://www.rbqueimaduras.com.br

Brasil. (2012). Ministério da Saúde. Indicadores para a saúde no Brasil: conceitos e aplicações. Brasília: ministério da saúde. p. 299. hhttp://datasus.gov.br.

Morais, T. O., Brito, C. C., Boulhosa, F. J. S., Ramos, L. N. C., Cordeiro, R. M., Esteves, P. K. S., Gazel, P. P., Martins, G. L. (2015). Fisioterapia em grande queimado: relato de caso em centro de tratamento de queimados na amazonia brasileira. Rev Bras Queimaduras. 2015;14(4):285-9 http://www.rbqueimaduras.com.br

Comini, A., Lança, P., Antunes. R., Franco, F. O. J., Veronesi, S. P., Alves, M. V., Sanches, J. A. \& Ramos, G. (2017). Perfil epidemiológico dos pacientes idosos queimados internados em unidade de tratamento de queimados do noroeste paulista. Rev Bras Queimaduras, 16(2):76-80 http://www.rbqueimaduras.com.br

Dutra, J. P. S., Custodio, S. R., Piccolo, N. \& Piccolo, R. D. (2017). Estudo clinico-epidemiologico de pacientes queimados internados em uma unidade de terapia intensiva em goiás. Rev Bras Queimaduras. 2017;16(2):87-93 http://www.rbqueimaduras.com.br

Ferreira, T. S., Lima, L. D. O., Anjos, R. N., Canuto, J. P., Marques, M. P. D. \& Santos, M. K. L. (2020). Dificuldades, complicações e relações interpessoais na assistência ao paciente queimado: uma abordagem sobre discursos. Reseach, Society and Development, 9(9) http://dx.doi.org/10.33448/rsd-v9i9.7693

Fernandes, M. I. S. (2019). Atuação da fisioterapia dermatofuncional na reabilitação de pacientes queimados: uma revisão integrativa de literatura. Rev Uningá, 56(3). http://revista.uninga.br/

Giordani, A. T., Sonobe, H. M., Guarini, G. \& Stadler, D. (2016). Complicações em pacientes queimados: revisão integrativa. Rev Eletrônica Gest Saúde. 7(2). https://dialnet.unirioja 
Research, Society and Development, v. 10, n. 15, e52101522478, 2021

(CC BY 4.0) | ISSN 2525-3409 | DOI: http://dx.doi.org/10.33448/rsd-v10i15.22478

Giuli, A. E., Itakussu, E. I., Valenciano, P. J., Shizuko, D. F. \& Salmaso, C. T. (2015). Caracterizaçâo de idosos vitimas de queimaduras internados em um centro de treinamento de queimados. Rev Bras Queimaduras. 14(4):253-6 http://www.rbqueimaduras.com.br

Villanova, M. V., Kenji, E. A. \& Costa, A. F. F. (2017). Guyton e Hall fundamentos da fisiologia / John E. Hall, Arthur C. Guyton; (13a ed.), Elsevier

Sakai, R. L., Matos, F. M. B., Wada, A., Sanches, D. C. P., Mattar, C. A., Cavalcante, P. C. A. \& Leão, F. (2012). Enxerto de pele parcial para tratamento de sequela de queimadura cáustica em cavidade oral: relato de caso. Rev Bras Queimaduras. 11(1):47-50 http://www.rbqueimaduras.com.br/

Santiago, A., freitas, C. \& Melo, M. (2018). Queimaduras, sequelas e tratamento fisioterapêutico: uma revisão de literatura. Revista Interfaces da Saúde $\cdot$. 3845. https://www.fvj.br/revista

Souza, S. \& Amorim, C. (2015). Queimadura panfacial: relato de caso. Rev Bras Queimaduras. 14(4):279-84. http://www.rbqueimaduras.com.br

Santana, C., Brito, C. \& Costa, A. (2012). Importancia da fisioterapia na reabilitaçao do paciente queimado. Rev Bras Queimaduras. 11(4):240-5. http://www.rbqueimaduras.com.br/

Santos, G., Freitas, N., Bastos, V. D. \& Feitosa, F. C. (2017). Perfil epidemiologico do adulto internado em um centro de referencia em tratamento de queimaduras. Rev Bras Queimaduras. 16(2):81-6 http://www.rbqueimaduras.com.br/

Felix, L. C. S., Galletti, M. I. S. \& Reis, T. C. F. (2014). Abordagem fisioterapêutica em queimados: revisão sistemática. Centro Universitário do Pará CESUPA. - Revista da Universidade Vale do Rio Verde, Três Corações, 12(2), 821-830.

Queiroz, R. C. P. F., Souza, R. F., Silveira, M. S. N., Barros, D. P., Postalli, T. J. P., Tavares, S. S., Alves, M. F. \& Gomes, D. B. (2015). Perfil dos pacientes evasores atendidos no ambulatório de queimados do conjunto hospitalar de Sorocaba. Rev. Bras. Queimaduras: 14 (4): $263-26$. http://www.rbqueimaduras.com.br/

Siqueira, F. M. B. \& Juliboni, E. P. K. (2012). O papel da atividade fisioterapêutica na reabilitação do individuo em fase aguda. http://www.cadernosdeterapiaocupacional.ufscar.br/

Lopes, J. R. J., Albuquerque, N. N. B. \& Silva, P. A. C. (2014). Terapia ocupacional em queimados: pesquisa bibliográfica acerca da reabilitação física junto a indivíduos com queimaduras. Rev Bras Queimaduras. 13(1):11-7. http://www.rbqueimaduras.com.br/

Santos, F., Geraldini, P. B. M., Ostrowski, V. R., Tezza, R. \& Silva, J. D. (2015). Relação entre mobilização precoce e tempo de internação em uma unidade de terapia intensiva. Revista Eletrônica Gestão \& Saúde. 06(2), 1394-07.

Wylker, J. \& Nunes, J. (2012). Assistência de enfermagem em grupos de riscos a queimadura. Rev Bras Queimaduras. 11(1):31-7. http://www.rbqueimaduras.org.br/. 